\title{
ON SOME MISCONCEPTIONS AND CHATTERJEE-TYPE $G$-CONTRACTION
}

\author{
T. Phaneendra ${ }^{1}$, S. Saravanan ${ }^{2}$ \\ ${ }^{1,2}$ Department of Mathematics \\ School of Advanced Sciences, \\ VIT University \\ Vellore-632014, Tamil Nadu, INDIA
}

\begin{abstract}
Let $(X, d)$ be a $G$-metric space, $f$, a self-map on $X$ and $x_{0} \in X$. Some misconceptions are brought about in findings of Mustafa et al [2], and a fixed point theorem for a Chatterjee-type $G$-contraction on a complete $G$-metric space is proved. More over, the unique fixed point $\mathrm{p}$ will be its contractive fixed point, in the sense that for each each $x_{0} \in X$, the $f$-iterates $x_{0}, f x_{0}, \ldots, f^{n} x_{0}, \ldots$ converge to $p$.
\end{abstract}

AMS Subject Classification: $54 \mathrm{H} 25$

Key Words: $G$-metric space, Chatterjee-type $G$-contraction, fixed point, $G$-contractive fixed point

\section{Introduction}

Let $X$ be a nonempty set and $G: X \times X \times X \rightarrow \mathbb{R}$ such that

(G1) $G(x, y, z) \geq 0$ for all $x, y, z \in X$ with $G(x, y, z)=0$ if $x=y=z$,

(G2) $G(x, x, y)>0$ for all $x, y \in X$ with $x \neq y$,

(G3) $G(x, x, y) \leq G(x, y, z)$ for all $x, y, z \in X$ with $z \neq y$,

Received:

Revised:

Published:

September 13, 2016

October 5, 2016

${ }^{\S}$ Correspondence author (c) 2016 Academic Publications, Ltd. url: www.acadpubl.eu 
(G4) $G(x, y, z)=G(x, z, y)=G(y, x, z)=G(z, x, y)$ $=G(y, z, x)=G(z, y, x)$ for all $x, y, z \in X$

(G5) $G(x, y, z) \leq G(x, w, w)+G(w, y, z)$ for all $x, y, z, w \in X$

Then $G$ is called a $G$-metric on $X$ and the pair $(X, G)$, a $G$-metric space. Axiom (G4) reveals that $G$ is symmetric in the three variables $x, y$ and $z$, and Axiom (G5) is referred to as the rectangle inequality (of $G$ ). This notion was introduced by Mustafa and Sims [3] in 2006.

From the definition of $G$-metric space, it immediately follows that

$$
G(x, y, y) \leq 2 G(x, x, y) \text { for all } x, y \in X
$$

We use the following notions, developed in [3]:

Definition 1.1. Let $(X, G)$ be a $G$-metric space. A $G$-ball in $X$ is defined by

$$
B_{G}(x, r)=\{y \in X: G(x, y, y)<r\} .
$$

It is easy to see that the family of all $G$-balls forms a base topology, called the $G$-metric topology $\tau(G)$ on $X$.

Also

$$
\rho_{G}(x, y)=G(x, y, y)+G(x, x, y) \text { for all } x, y \in X
$$

induces a metric on $X$, and the $G$-metric topology coincides with the metric topology induced by the metric $\rho_{G}$. This allows us to readily transform many concepts from metric space into the setting of $G$-metric space.

Definition 1.2. A sequence $\left\langle x_{n}\right\rangle_{n=1}^{\infty}$ in a $G$-metric space $(X, G)$ is said to be $G$-convergent with limit $p \in X$ if it converges to $p$ in the $G$-metric topology $\tau(G)$.

Lemma 1.1. The following statements are equivalent in a $G$-metric space $(X, G)$ :

(a) $\left\langle x_{n}\right\rangle_{n=1}^{\infty} \subset X$ is G-convergent with limit $p \in X$,

(b) $\lim _{n \rightarrow \infty} G\left(x_{n}, x_{n}, p\right)=0$,

(c) $\lim _{n \rightarrow \infty} G\left(x_{n}, p, p\right)=0$.

Definition 1.3. A sequence $\left\langle x_{n}\right\rangle_{n=1}^{\infty}$ in a $G$-metric space $(X, G)$ is said to be $G$-Cauchy if $\lim _{n, m \rightarrow \infty} G\left(x_{n}, x_{m}, x_{m}\right)=0$. 
Definition 1.4. A $G$-metric space $(X, G)$ is said to be $G$-complete if every $G$-Cauchy sequence in $X$ converges in it.

Definition 1.5. Let $(X, G)$ be a $G$-metric space. A set $S \subset X$ is said to be $G$-bounded or simply bounded if there exists a positive number $M$ such that $G(x, y, z)<M$ for all $x, y, z \in S$.

Definition 1.6. Let $(X, G)$ be a $G$-metric space. We define the diameter of $S \subset X$ by $\operatorname{diam} S=\delta(S)=\sup \{G(x, y, z): x, y, z \in S\}$. The set $S$ is $G$-bounded if and only if $\delta(S)<\infty$.

As a part of an extensive research in $G$-metric spaces, we refer to a couple of interesting results from [2]. The first of them is:

Theorem 1.1. Let $(X, G)$ be a complete $G$-metric space and $f: X \rightarrow X$ satisfying one of the following conditions:

$$
G(f x, f y, f y) \leq k \max \{G(x, f y, f y), G(y, f x, f x), G(y, f y, f y)\}
$$

or

$$
G(f x, f y, f y) \leq k \max \{G(x, x, f y), G(y, y, f x), G(y, y, f y)\}
$$

for all $x, y, z \in X$, where $0 \leq k<1$. Then $f$ has a unique fixed point $p$ and $f$ is $G$-continuous at $p$.

In the proof of Theorem 1.1, the authors used the following notations:

$$
\begin{aligned}
\Gamma_{n} & =\max \left\{G\left(x_{i}, x_{j}, x_{j}\right): i, j \in\{0,1, \ldots, n+1\}\right\}, n \in \mathbb{N} \\
\Gamma & =\max \left\{\Gamma_{k}: k=n, \ldots, m-1\right\} \text { for } m>n,
\end{aligned}
$$

where $x_{n}=f^{n} x_{0}$ for each $x_{0} \in X$. The authors used the induction to show that

$$
G\left(x_{n}, x_{n+1}, x_{n+1}\right) \leq k^{n} \Gamma_{n} .
$$

Then with $\Gamma=\max \left\{\Gamma_{k}: k=n, \ldots, m-1\right\}$ for $m>n$, and the rectangle inequality, they established

$$
G\left(x_{n}, x_{m}, x_{m}\right) \leq\left(\frac{k^{n}}{1-k}\right) \Gamma .
$$

Further, the authors employed the limit as $n \rightarrow \infty$ in (6) to see that

$$
G\left(x_{n}, x_{m}, x_{m}\right) \rightarrow 0 .
$$


That is $\left\langle x_{n}\right\rangle_{n=1}^{\infty}$ is a Cauchy sequence.

We have two observations in these arguments. Firstly, the sets

$$
A_{n}=\left\{G\left(x_{i}, x_{j}, x_{j}\right): i, j \in\{0,1, \ldots, n+1\}\right\}, n=1,2,3, \ldots
$$

constitute an expanding sequence of sets of nonnegative real numbers. Hence

$$
\max A_{n} \leq \max A_{n+1} \text { or } \Gamma_{n} \leq \Gamma_{n+1} \text { for all } n .
$$

Therefore, $\Gamma=\max \left\{\Gamma_{n}, \Gamma_{n+1}, \ldots, \Gamma_{m-1}\right\}=\Gamma_{m-1}$.

Then for $m>n$, from the repeated application of the rectangle inequality and (5), it follows that

$$
\begin{aligned}
& G\left(x_{n}, x_{m}, x_{m}\right) \\
& \quad \leq \underbrace{G\left(x_{n}, x_{n+1}, x_{n+1}\right)+G\left(x_{n+1}, x_{n+2}, x_{n+2}\right)+\ldots+G\left(x_{m-1}, x_{m}, x_{m}\right)}_{m-n \text { terms }} \\
& \quad \leq k^{n}\left(1+k+\cdots+k^{m-n-1}\right) \Gamma_{m-1} \leq\left(\frac{k^{n}}{1-k}\right) \Gamma_{m-1},
\end{aligned}
$$

which gives (6).

Definition 1.7. Given $x_{0} \in X$, the orbit at $x_{0}$ is the the sequence of iterates

$$
O_{f}\left(x_{0}\right)=\left\{x_{0}, x_{1}, \ldots, x_{n}, \ldots\right\}, \text { where } x_{n}=f x_{n-1} \text { for } n \geq 1 .
$$

Remark 1.1. We now claim that the above proof of (7) requires that $O_{f}\left(x_{0}\right)$ at each $x_{0} \in X$ is bounded so that $\sup \left[O_{f}\left(x_{0}\right)\right]=\delta<\infty$.

If possible, suppose that $O_{f}\left(x_{0}\right)$ is unbounded. Then there exists a positive integer $n$ such that

$$
G\left(x_{1}, x_{n}, x_{n}\right) \geq \mu \max \left\{G\left(x_{1}, x_{r}, x_{r}\right): 0 \leq r \leq n-1\right\},
$$

where

$$
\mu=\max \left\{\frac{2 k}{1-k}, 2 k\right\} .
$$

Now from the inequality (3), we have

$$
G\left(x_{1}, x_{n}, x_{n}\right) \leq k \max \left\{G\left(x_{0}, x_{n}, x_{n}\right), G\left(x_{n-1}, x_{1}, x_{1}\right), G\left(x_{n-1}, x_{n}, x_{n}\right)\right\} .
$$

Here $M=\max \left\{G\left(x_{0}, x_{n}, x_{n}\right), G\left(x_{n-1}, x_{1}, x_{1}\right), G\left(x_{n-1}, x_{n}, x_{n}\right)\right\}$. 
Case(a): Suppose that $M=G\left(x_{0}, x_{n}, x_{n}\right)$. Then (13) gives

$$
\begin{aligned}
G\left(x_{1}, x_{n}, x_{n}\right) & \leq k G\left(x_{0}, x_{n}, x_{n}\right) \\
& \leq k\left[G\left(x_{0}, x_{1}, x_{1}\right)+G\left(x_{1}, x_{n}, x_{n}\right)\right] \\
& \leq\left(\frac{k}{1-k}\right) G\left(x_{0}, x_{1}, x_{1}\right) \\
& \leq \mu \max \left\{G\left(x_{0}, x_{r}, x_{r}\right): 0 \leq r \leq n\right\} \\
& <G\left(x_{1}, x_{n}, x_{n}\right)
\end{aligned}
$$

which is a contradiction.

Case(b): Suppose that $M=G\left(x_{n-1}, x_{1}, x_{1}\right)$. Then (13) gives

$$
\begin{aligned}
G\left(x_{1}, x_{n}, x_{n}\right) & \leq k G\left(x_{n-1}, x_{1}, x_{1}\right) \leq 2 k G\left(x_{1}, x_{n-1}, x_{n-1}\right) \\
& \leq \mu \max \left\{G\left(x_{0}, x_{r}, x_{r}\right): 0 \leq r \leq n\right\}<G\left(x_{1}, x_{n}, x_{n}\right)
\end{aligned}
$$

which is again a contradiction.

Case(c): Finally, suppose that $M=G\left(x_{n-1}, x_{n}, x_{n}\right)$. Then (13) gives

$$
\begin{aligned}
G\left(x_{1}, x_{n}, x_{n}\right) & \leq k G\left(x_{n-1}, x_{n}, x_{n}\right) \\
& \leq k\left[G\left(x_{n-1}, x_{1}, x_{1}\right)+G\left(x_{1}, x_{n}, x_{n}\right)\right. \\
& \leq\left(\frac{2 k}{1-k}\right) G\left(x_{1}, x_{n-1}, x_{n-1}\right) \\
& \leq \mu \max \left\{G\left(x_{0}, x_{r}, x_{r}\right): 0 \leq r \leq n\right\} \\
& <G\left(x_{1}, x_{n}, x_{n}\right)
\end{aligned}
$$

which is also a contradiction.

These three contradictions prove that $O_{f}\left(x_{0}\right)$ is bounded and $\sup \left[O_{f}\left(x_{0}\right)\right]=$ $\delta<\infty$.

Then from (6), it follows that

$$
G\left(x_{n}, x_{m}, x_{m}\right) \leq \frac{k^{n} \delta}{1-k}
$$

Applying the limit as $n \rightarrow \infty$ in (14), we get (7). This proves that $\left\langle x_{n}\right\rangle_{n=1}^{\infty}$ is a Cauchy sequence.

The second result of [2] is: 
Theorem 1.2. Let $(X, G)$ be a complete $G$-metric space and $f: X \rightarrow X$ satisfying one of the following conditions:

$$
\begin{gathered}
G(f x, f y, f z) \leq k \max \{G(x, f y, f y), G(x, f z, f z), G(y, f x, f x) \\
G(y, f z, f z), G(z, f x, f x), G(z, f y, f y)\}
\end{gathered}
$$

or

$$
\begin{gathered}
G(f x, f y, f z) \leq k \max \{G(x, x, f y), G(x, x, f z), G(y, y, f x) \\
G(y, y, f z), G(z, z, f x), G(z, z, f y)\}
\end{gathered}
$$

for all $x, y, z \in X$, where $0 \leq k<1$. Then $f$ has a unique fixed point $p$ and $f$ is $G$-continuous at $p$.

Remark 1.2. It was claimed in [2] that Theorem 1.2 is a Corollary to Theorem 1.1, which is a misconception. In fact, the conditions (3) and (4) follow as particular cases of conditions (15) and (16) with $y=z$ respectively. Therefore, it is appropriate to assert that Theorem 1.1 is a Corollary to Theorem 1.2. The proof for Theorem 1.2 is just similar to the above proof and is omitted here.

Remark 1.3. Also if $k=0$, writing $z=y=f x$ in (15) or (16), we see that $G\left(f x, f^{2} x, f^{2} x\right)=0$ so that $f^{2} x=f x$ for each $x \in X$. That is, every $f x$ is a fixed point of $f$ Theorem 1.2. In other words, the fixed point is not unique in Theorem 1.2. Therefore, Theorem 1.2 in its revised form is stated as follows:

Theorem 1.3. Let $(X, G)$ be a $G$-metric space and $f: X \rightarrow X$ satisfying either (15) or (16), where $0<k<1$. If $X$ is $G$-complete, then $f$ has a unique fixed point $p$.

Omitting the terms $G(x, f z, f z), G(y, f x, f x)$ and $G(z, f y, f y)$ in (15), and restricting $k$ to $(0,1 / 3)$, say $0 \leq \gamma<1 / 3$, we get

Corollary 1.1. Let $(X, G)$ be a complete $G$-metric space and $f: X \rightarrow X$ satisfying one of the following conditions:

$$
\begin{gathered}
G(f x, f y, f z) \leq \gamma \max \{G(x, f y, f y), G(y, f z, f z), G(z, f x, f x)\} \\
\text { for all } x, y, z \in X,
\end{gathered}
$$

where $0 \leq \gamma<1 / 3$. Then $f$ has a unique fixed point.

Since the maximum of three nonnegative numbers cannot exceed their sum, (17) is weakened as

$$
G(f x, f y, f z) \leq \gamma[G(x, f y, f y)+G(y, f z, f z)+G(z, f x, f x)]
$$




$$
\text { for all } x, y, z \in X \text {, }
$$

where $0<\gamma<1 / 3$. This is analogous to Chatterjee's contraction [1] in metric space with the choice

$$
\rho(f x, f y) \leq c[G(x, f y)+G(y, f x)] \text { for all } x, y \in X
$$

where $0<c<1 / 2$. We therefore call $f$ satisfying (18), a Chatterjee-type $G$-contraction.

In the next section, we shall obtain a fixed point for a Chatterjee-type $G$-contraction on a complete $G$-metric space.

\section{Main Result}

The notion of $G$-contractive fixed point was introduced by Phaneendra with Kumara Swamy in [4]. In fact

Definition 2.1. A fixed point $p$ of $f$ on a $G$-metric space $(X, G)$ is a $G$-contractive fixed point of it if the orbit $O_{f}\left(x_{0}\right)=\left\langle x_{0}, f x_{0}, \ldots, f^{n} x_{0}, \ldots\right\rangle$ at each $x_{0} \in X$ is $G$-convergent with limit $p$.

It was shown that the unique fixed point of the self-map $f$ with the following choices is a $G$-contractive fixed point.

(a) $G(f x, f y, f z) \leq q G(x, y, z)$ for all $x, y, z \in X$, where $0 \leq q<1$,

(b) $G(f x, f y, f z) \leq a G(x, f x, f x)+b G(y, f y, f y)+c G(z, f z, f z)+e G(x, y, z)$ for all $x, y, z \in X$, where $a, b, c$ and $e$ are nonnegative real numbers with $a+b+c+e<1$.

We now prove

Theorem 2.1. Let $f$ be a Chatterjee-type contraction on a complete Gmetric space $(X, G)$ with the choice (18). Then $f$ has a unique fixed point $p$, which will be its contractive fixed point as well.

Proof. Let $x_{0} \in X$ be arbitrary. Define $\left\langle x_{n}\right\rangle_{n=1}^{\infty} \subset X$ by

$$
x_{n}=f x_{n-1} \text { for } n \geq 1 \text {. }
$$

Writing $x=x_{n-1}$ and $y=z=x_{n}$ in (18) and then using (20) and (G5), we get

$$
G\left(f x_{n-1}, f x_{n}, f x_{n}\right)=G\left(x_{n}, x_{n+1}, x_{n+1}\right)
$$




$$
\begin{aligned}
& \leq \gamma\left[G\left(x_{n-1}, f x_{n}, f x_{n}\right)+G\left(x_{n}, f x_{n}, f x_{n}\right)+G\left(x_{n}, f x_{n-1}, f x_{n-1}\right)\right] \\
& \leq \gamma\left[G\left(x_{n-1}, x_{n+1}, x_{n+1}\right)+G\left(x_{n}, x_{n+1}, x_{n+1}\right)+G\left(x_{n}, x_{n}, x_{n}\right)\right] \\
& \leq \gamma\left[G\left(x_{n-1}, x_{n}, x_{n}\right)+2 G\left(x_{n}, x_{n+1}, x_{n+1}\right)\right] \\
& \leq k G\left(x_{n-1}, x_{n}, x_{n}\right),
\end{aligned}
$$

where $k=\frac{\gamma}{1-2 \gamma} \cdot$ By induction, we have

$$
G\left(x_{n}, x_{n+1}, x_{n+1}\right) \leq k^{n} G\left(x_{0}, x_{1}, x_{1}\right) \text { for } n \geq 1 .
$$

Now for all $n, m \in N$ with $m>n$, by $(G 5)$ and (21), we obtain

$$
\begin{aligned}
G\left(x_{n}, x_{m}, x_{m}\right) \leq & G\left(x_{n}, x_{n+1}, x_{n+1}\right)+G\left(x_{n+1}, x_{n+2}, x_{n+2}\right) \\
& +\cdots+G\left(x_{m-1}, x_{m}, x_{m}\right)(m-n \text { terms }) \\
& \leq(\underbrace{k^{n}+k^{n+1}+k^{n+2}+\cdots+k^{n+(m-n-1)}}_{m-n \text { terms }}) G\left(x_{0}, x_{1}, x_{1}\right) \\
& =k^{n}(\underbrace{1+k+k^{2}+\cdots+k^{m-n-1}}_{m-n \text { terms }}) G\left(x_{0}, x_{1}, x_{1}\right) \\
& \leq k^{n} \cdot \frac{1-k^{m-n}}{1-k} \cdot G\left(x_{0}, x_{1}, x_{1}\right) \\
& \leq \frac{k^{n}}{1-k} \cdot G\left(x_{0}, x_{1}, x_{1}\right) .
\end{aligned}
$$

Since $k=\frac{\gamma}{1-2 \gamma}<1$, applying the limit as $n \rightarrow \infty$ in this, we find that $\lim _{n, m \rightarrow \infty} G\left(x_{n}, x_{m}, x_{m}\right)=0$.

Thus $\left\langle x_{n}\right\rangle_{n=1}^{\infty}$ is $G$-Cauchy sequence in $X$. Since $X$ is $G$-Complete, there exists a point $p \in X$ such that $\left\langle x_{n}\right\rangle_{n=1}^{\infty}$ is $G$-convergent to $p$. That is

$$
\lim _{n \rightarrow \infty} x_{n-1}=\lim _{n \rightarrow \infty} x_{n}=p
$$

Now writing $x=x_{n-1}$ and $y=z=p$ in (18),

$$
\begin{aligned}
G\left(f x_{n-1},\right. & f p, f p)=G\left(x_{n}, f p, f p\right) \\
& \leq \gamma\left[G\left(x_{n-1}, f p, f p\right)+G(p, f p, f p)+G\left(p, f x_{n-1}, f x_{n-1}\right)\right] \\
& \leq \gamma\left[G\left(x_{n-1}, f p, f p\right)+G(p, f p, f p)+G\left(p, x_{n}, x_{n}\right)\right]
\end{aligned}
$$

Proceeding the limit as $n \rightarrow \infty$ in this and using (22), and then simplifying, we get

$$
G(p, f p, f p) \leq 2 \gamma G(p, f p, f p)
$$


If $f p \neq p,(23)$ would imply that

$$
0<G(p, f p, f p) \leq 2 \gamma G(p, f p, f p)<G(p, f p, f p),
$$

which is a contradiction. Therefore, $f p=p$. That is $p$ is a fixed point of $f$. The uniqueness of the fixed point follows easily from (18).

We finally prove that $p$ is a G-Contractive fixed point of $f$. In fact, let $x_{0} \in X$ be arbitrary. Writing $x=f^{n-1} x_{0}$ and $y=z=p$ in (18) and using (G5), we get

$$
\begin{aligned}
G\left(f^{n} x_{0}, p, p\right) & =G\left(f^{n} x_{0}, f p, f p\right) \\
& \leq \gamma\left[G\left(f^{n-1} x_{0}, f p, f p\right)+G(p, f p, f p)+G\left(p, f^{n} x_{0}, f^{n} x\right)\right] \\
& \leq \gamma\left[G\left(f^{n-1} x_{0}, p, p\right)+2 G\left(f^{n} x_{0}, p, p\right)\right] \\
& \leq \frac{\gamma}{1-2 \gamma} G\left(f^{n-1} x_{0}, p, p\right) .
\end{aligned}
$$

Since $\frac{\gamma}{1-2 \gamma}<1$, we see that $G\left(f^{n} x_{0}, p, p\right) \rightarrow 0$ as $n \rightarrow \infty$ for each $x_{0} \in X$. Thus $p$ is a $G$-Contractive fixed point of $f$.

\section{Conclusion}

Let $(X, d)$ be a $G$-metric space, $f$, a self-map on $X$ and $x_{0} \in X$. Misconceptions regarding two fixed point theorems of Mustafa et al [2] have been discussed. Then a fixed point theorem for a Chatterjee-type $G$-contraction on a complete $G$-metric space has been proved. The unique fixed point will be its contractive fixed point, to which the $f$-iterates $x_{0}, f x_{0}, \ldots, f^{n} x_{0}, \ldots$ converge, for each each $x_{0} \in X$.

\section{Acknowledgments}

The authors are thankful for the referees for their valuable suggestions in finalizing the paper.

\section{References}

[1] S.K. Chatterjea, Fixed-point theorems, C.R. Acad. Bulgare Sci., 25 (1972), 727-730, MR $48 \# 2845$. 
[2] Z. Mustafa, H. Obiedat, F. Awawdeh, Some fixed point theorems for mapping on complete G-Metric Spaces, Fixed Point Theory and Applications (2008), Article ID 189870, 1-12, doi: $10.1155 / 2008 / 189870$.

[3] Z. Mustafa, B. Sims, A new approach to generalized metric spaces, Jour. Nonlinear and Convex Anal., 7, No. 2 (2006), 289-297.

[4] T. Phaneendra, K. Kumara Swamy, Unique fixed point in G-metric space through greatest lower bound properties, Novi Sad J. Math., 43 (2013), 107-115. 